\title{
COMPORTEMENT DES NOUVEAUX RIZ AFRICAINS FACE A LA PYRICULARIOSE EN COTE D'IVOIRE : CAS DU NERICA 1 (BONFANI) ET DU NERICA 2 (KEAH).
}

\author{
A. BOUET ${ }^{1}$, H. ADREIT ${ }^{2}$, J. MILLAZO², D. THARREAU ${ }^{2}$ et J. Z. KELI' \\ ${ }^{1}$ Centre National de Recherche Agronomique (C.N.R.A.), BP 440 Man, Côte d'Ivoire \\ ${ }^{2}$ Centre de Coopération Internationale en Recherche Agronomique pour le Développement, \\ Département des cultures annuelles (C.R.A.D - C.A), campus international de baillarguet 34398 Montpellier Cedex 5
}

\begin{abstract}
RESUME
Les variétés de riz pluvial (oryza glaberrima x Oryza sativa) NERICA1 (Bonfani) et NERICA2 (Kéah), grâce à leur potentiel agronomique, sont en diffusion en Côte d'Ivoire depuis près de 3 ans. Cela, dans un contexte épidémique marqué par une pression relativement forte de la pyriculariose maladie fongique due à Magnaporthe grisea (Barr, 1977), très préjudiciable au riz Oriza sativa. Dans le but de prévenir d'éventuelles attaques sévères des NERICA par la maladie, une étude d'évaluation de leur résistance a été conduite en condition contrôlée. Elle a consisté à confronter par inoculation au stade végétatif (4-5 feuilles), ces variétés de riz à 40 isolats de Magnaporthe grisea, représentatifs de la diversité au sein de la population du parasite en Côte d'Ivoire. Les résultats des inoculations ont montré une résistance, relativement générale chez NERICA 2 à la pyriculariose, contrairement à NERICA 1 qui a été sévèrement attaqué par certaines souches de la maladie. Ces informations recommandent, au niveau des stratégies de lutte contre la pyriculariose, de privilégier dans les zones à risque, la culture de NERICA 2 au NERICA 1 ou, de cultiver cette dernière variété soit en association, soit en rotation avec le NERICA 2. Par ailleurs, il sera opportun d'apprécier la réaction du NERICA 1 et du NERICA 2 à la pyriculariose du cou qui à l'instar de la pyriculariose foliaire, engendre des pertes de récolte souvent importantes sur le riz en Côte d'lvoire.
\end{abstract}

Mots clés : Riz, Variétés NERICA, Pyriculariose, résistance, inoculation, Côte d'Ivoire.

\section{ABSTRACT}

REACTION OF NEW AFRICA RICE TO BLAST IN COTE D'IVOIRE : CASE OF NERICA 1 (BONFANI) AND NERICA 2 (KEAH)

NERICA 1 (Bonfani) and NERICA 2 (Kéah) are two upland rice (oryza glaberrima $x$ Oryza sativa) varieties characterized by high yield potentials. For the past three years, these varieties have been extensively used in rice growing regions of Côte d'Ivoire. Unfortunately, the crop has been subject to omprtant fungus attack. The rice Oriza sativa blast, is caused by a fungus, known as Magnaporthe grisea (Barr, 1977). In order to assess the incidence of the disease on these varieties, an inoculation trial involving 40 isolates, of the pathogen was conducted. NERICA 2 exhibited foliar resistance to all the isolates whereas NERICA 1 was severely attacked. Besides the leaves, the pathogen also affects the neck of the plant which results in important yield losses. Therefore, it would be worthwhile determining the behaviour of the varieties to neck blast.

Key words : Riz, NERICA variety, blast, inoculation, resistance, Côte d'Ivoire.

\section{INTRODUCTION}

Les nouveaux riz africains ou NERICA (New Rice for Africa) sont des hybrides interspécifiques (Oryza glaberrima $X$ O. sativa) mises au point par l'ADRAO (actuellement dénommé Centre Africain pour le Riz) dans les années 90 (Anonyme 1, 1998). Ces variétés sont considérées par des agronomes comme des moteurs de la prochaine révolution verte en Afrique.

En effet, concernant les NERICA 1 et 2, homologués pour la riziculture pluviale en Côte d'Ivoire sous les noms respectifs de Bonfani et de Kéah, les rendements annoncés sont très supérieurs à la moyenne nationale. Cela, dans 
un contexte général d'agriculture non intensive. En plus de leur performance agronomique (Dingkuhn et al., 1997 ; Anonyme 2, 2001), ces NERICA sont présentés comme étant résistants à des contraintes biotiques (Plowright et al., 1999 ; Johnson et al., 1998) dont la pyriculariose demeure la plus préjudiciable au riz pluvial en Côte d'Ivoire.

Fort de ces atouts agronomiques que l'on leur attribue, le NERICA 1 et le NERICA 2 sont en très large diffusion à travers des projets tels que le CBSS (Communauty Based seed System ou Système de Production de semences Communautaires), le PADS (Projet d'Adaptation et de Diffusion de technologies des systèmes à base spécifique) et « riz pour tous «. Cependant, une question majeure reste à élucider même si elle paraît tardive ; il s'agit en effet de savoir si les variétés Bonfani et le Kéah peuvent résister durablement aux différentes sources de virulence de Magnaporthe grisea (champignon responsable de la pyriculariose) mises en évidence en Côte d'Ivoire (Bouet et al., 2002).

L'objectif principal de cette interrogation est d'évaluer le comportement de ces riz en vue de prévenir une éventuelle catastrophe agronomique pouvant être liée à une sévère attaque à l'échelle régionale ou nationale des variétés NERICA 1 et 2, par la pyriculariose. Aussi, sera-t-il possible de rationaliser la diffusion de ces nouveaux riz en Côte d'Ivoire.

\section{MATERIEL ET METHODES}

\section{MATERIEL BIOLOGIQUE}

Le matériel végétal utilisé a été constitué par les variétés de riz NERICA1 et NERICA 2. La variété Maratelli (sans gène de résistance connu à la pyriculariose) a été utilisée comme témoin de sensibilité. Le matériel fongique regroupait 40 isolats de $M$. grisea, représentatifs de la diversité au sein de la population du parasite en Côte d'Ivoire, notamment :

- 11 isolats extraits des prélèvements d'organes (feuilles et cous) de riz effectués en 2003 ;

- 09 isolats de l'ancienne collection (années 80) non encore étudiés ;

- 14 isolats issus des 3 groupes de races et des 5 lignées clonales révélés (Bouet et al., 2002);
- 06 isolats représentatifs des pathotypes des 20 isolats caractérisés en 2002 par Bouet (non publié).

\section{DISPOSITIF EXPERIMENTAL}

Les trois variétés de riz ont été semées chacune dans deux pots (diamètre $=7 \mathrm{~cm}$; profondeur $=$ $9 \mathrm{~cm}$ ) contenant du terreau comme substrat de culture. Au total, 120 pots contenant chacun 3 plantes par variété de riz ont été ensemencés. Pour prédisposer les plantes à l'infection par $M$. grisea, des apports d'urée à raison de 50 unités de N/ha ont été assurés chaque semaine et à la veille de l'inoculation.

L'inoculation a été faite par pulvérisation mécanique des feuilles (face supérieure du limbe), quand les plantes étaient au stade 4-5 feuilles (28 à 30 jours après semis). Chacun des 40 isolats de $M$. grisea a été inoculé 2 fois à chacune des 3 variétés étudiées. L'inoculum additionné de gélatine à $0,5 \%$ était dosé à 5 $10^{4}$ conidies $\mathrm{ml}^{-1}$.

Après l'inoculation, les plantes ont été placées à l'obscurité, dans un phytotron (Humidité relative $90 \%$; température $=22^{\circ} \mathrm{C}$ ). Elles en sont retirées $16 \mathrm{~h}$ après pour être exposées en serre à $27^{\circ} \mathrm{C}$ jusqu'à la notation des symptômes.

La sévérité de la pyriculariose foliaire a été notée 7 jours après l'inoculation selon une échelle graduée de 1 à 6 (Notteghem, 1981) et décrite comme suit :

1. pas de symptômes

2. lésions nécrotiques brunes de diamètre inférieur à $2 \mathrm{~mm}$

3. lésions nécrotiques à centre clair limité par une bordure nécrotique

4. lésions à centre grisâtre plus ou moins limitées

5. lésions à centre gris et à forme losangique typique

6. zones décolorées coalescentes non limitées par une bordure nécrotique

Un isolat est considéré comme avirulent quand les symptômes qu'il provoque sont notés 1, 2 ou 3. II est plus ou moins virulent quand les notes sont comprises entre 4 et 6 de l'échelle de notation. 


\section{RESULTATS}

\section{REACTIONS DE LAVARIETE NERICA 1}

Dans $70 \%$ des cas, les notes de sévérité de la pyriculariose oscillent entre 1 et 3 (tableau 1). Les lésions de type plus ou moins sensible (note : 4 à 5) ont été provoquées par les isolats CD17, CD16 d'Odienné, CD26 de Daoukro, CD47 de Boundiali, CD150, CD117 de Man, CD165 de Séguéla, CD203, CD208 de Tiassalé et CD122 de Waninou. II faut cependant remarquer les isolats CD150, CD203, CD208 et CD117 qui ont provoqué des lésions de type sensible (note : 5) sur le NERICA 1. La variété NERICA 1 est apparue ainsi résistante ( $R$ : note 1 à 3) ou moyennement résistante (MR : note de 4) à 30 des isolats étudiés. Au niveau géographique (Figure 1), les isolats virulents sur NERICA 1 sont relativement fréquents dans l'Ouest (Man), le Nord-ouest (Waninou, Touba, Odienné, Séguela, Boundiali) et dans le Sud (Tiassalé).

\section{REACTIONDELAVARIETENERICA2}

Excepté l'isolat CD47 qui a induit une note de sévérité égale à 4 sur la variété NERICA 2 , tous les autres se sont avérés avirulents sur le cultivar. Les notes qui attestent de cette résistance pratiquement générale de NERICA 2 sont comprises entre 1 et 3 (Tableau 1)

Tableau 1 : Réactions des variétés de riz NERICA 1 et NERICA 2 à 40 isolats de Magnaporthe grisea en Côte d'Ivoire.

Reaction of Nerica 1 and 2 rice varieties with respect to 40 isolates of Magnaporthe grisea in Côte d'lvoire

\begin{tabular}{|c|c|c|c|}
\hline \multirow[t]{2}{*}{ Isolat } & \multicolumn{2}{|c|}{$\begin{array}{l}\text { Note de sévérité } \\
\text { de la pyriculariose }\end{array}$} & \multirow{2}{*}{$\begin{array}{l}\text { Lieu de } \\
\text { collecte }\end{array}$} \\
\hline & NERICA 1 & NERICA 2 & \\
\hline CD 04 & 3 & 2 & Man \\
\hline CD 17 & 4 & 2 & Odienné \\
\hline CD 26 & 5 & 2 & Daoukro \\
\hline CD 47 & 5 & 4 & Boundiali \\
\hline CD 95 & 2 & 2 & Man \\
\hline CD 150 & 4 & 3 & Man \\
\hline CD 162 & 2 & 2 & Touba \\
\hline CD 165 & 5 & 3 & Séguéla \\
\hline CD 200 & 2 & 2 & Divo \\
\hline CD 201 & 2 & 2 & Oumé \\
\hline CD 116 & 2 & 2 & Man \\
\hline CD 202 & 2 & 2 & Tiassalé \\
\hline CD 203 & 5 & 3 & Tiassalé \\
\hline CD 204 & 2 & 2 & N'douci \\
\hline CD 205 & 2 & 2 & Issia \\
\hline CD 206 & 2 & 2 & Zéréguié \\
\hline CD 207 & 3 & 2 & Tiassalé \\
\hline CD 208 & 5 & 3 & Tiassalé \\
\hline CD 209 & 2 & 2 & Hermankono \\
\hline CD 210 & 2 & 2 & Tiassalé \\
\hline CD 16 & 4 & 3 & Odiénné \\
\hline CD 19 & 3 & 2 & Odiénné \\
\hline CD 42 & 3 & 3 & Bouaké \\
\hline CD 49 & 2 & 2 & Odiénné \\
\hline CD 65 & 2 & 2 & San-Pédro \\
\hline CD 69 & 2 & 2 & Bouaké \\
\hline CD 100 & 2 & 2 & Bouaké \\
\hline CD 113 & 2 & 2 & Man \\
\hline CD 117 & 5 & 3 & Man \\
\hline CD 120 & 2 & 2 & San-Pédro \\
\hline CD 122 & 4 & 2 & Waninou \\
\hline CD 123 & 2 & 2 & Sokourala \\
\hline CD 125 & 2 & 2 & Sokourala \\
\hline CD 126 & 3 & 2 & Farako \\
\hline CD 181 & 2 & 2 & Tchélélovogo \\
\hline CD 184 & 2 & 2 & Bouaké \\
\hline CD 190 & 2 & 2 & Bangolo \\
\hline CD 194 & 3 & 2 & Guiglo \\
\hline CD 196 & 2 & 1 & Man \\
\hline CD 199 & 2 & 2 & Guiglo \\
\hline
\end{tabular}




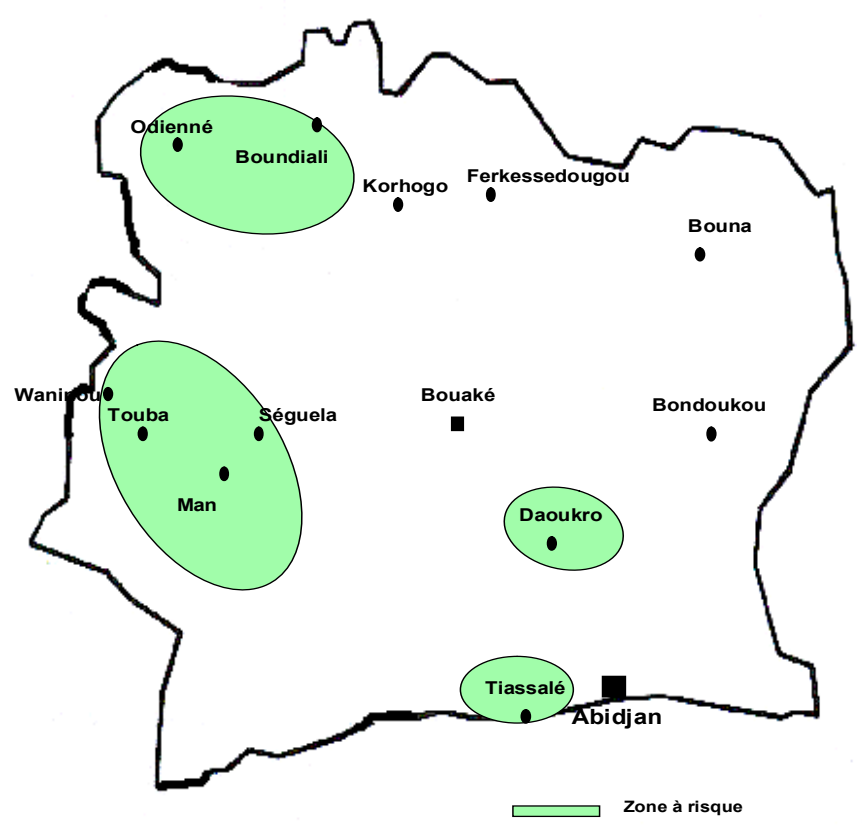

Figure 1 : Zone à risque élevé d'attaque du riz NERICA 1 par la pyriculariose en Côte d'Ivoire.

Area of high susceptibility of NERICA 1 rice to blast disease in Côte d'Ivoire.

\section{COMPARAISON DES REACTIONS DE NERICA 1 ET DE NERICA 2}

La figure 2 présente le niveau de résistance de chacune des deux variétés à l'ensemble des isolats inoculés. II en ressort que le NERICA 1 a un potentiel de résistance à la pyriculariose foliaire relativement inférieur à celui de NERICA 2 . En effet aucun des 40 isolats testés n'a engendré des lésions de types sensibles sur NERICA 2 alors que 10 d'entre eux sont relativement plus virulents sur NERICA 1 (Figure 3)

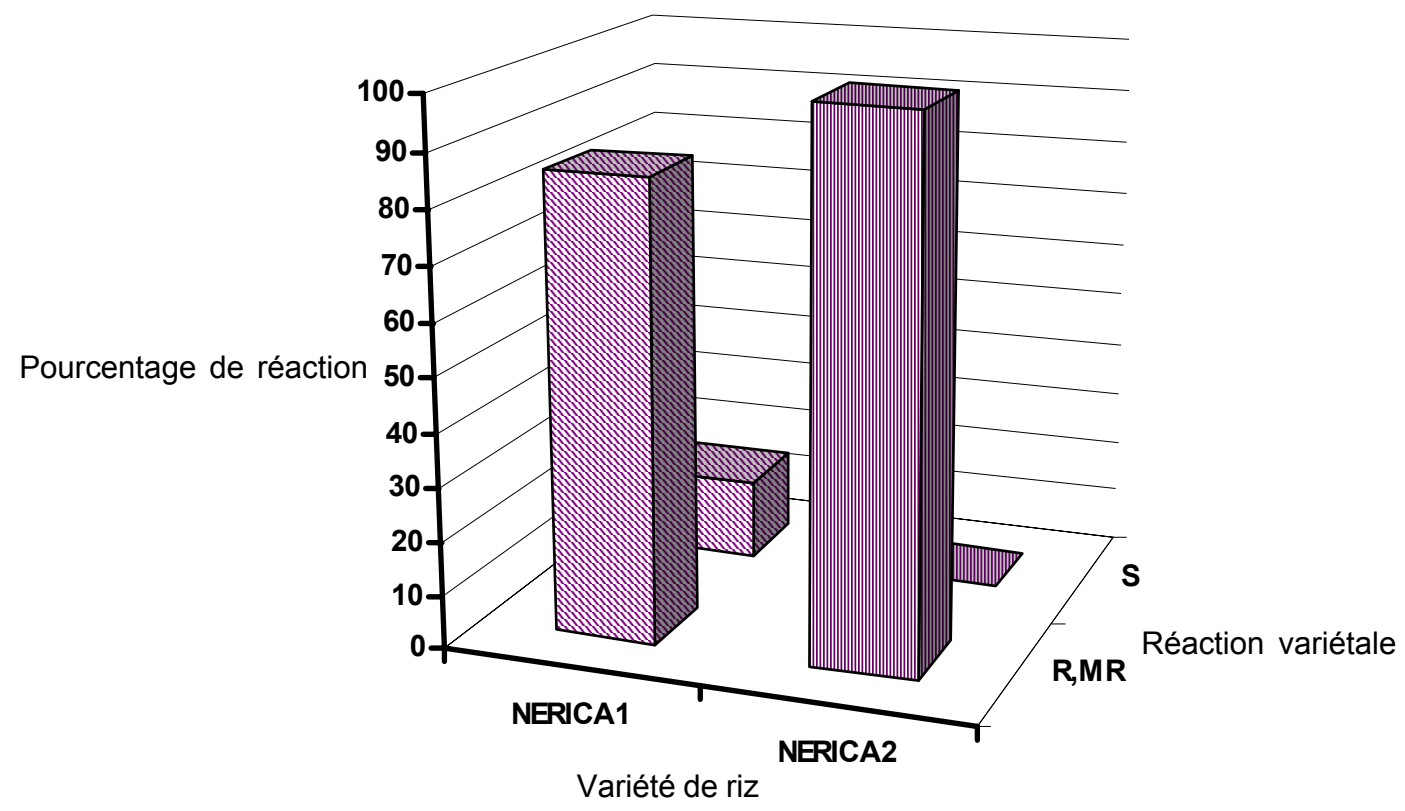

Figure 2 : Niveau de résistance des variétés de riz NERICA 1 et NERICA 2 à la pyriculariose Blast disease resistance level of rice varieties NERICA 1 and NERICA 2. 


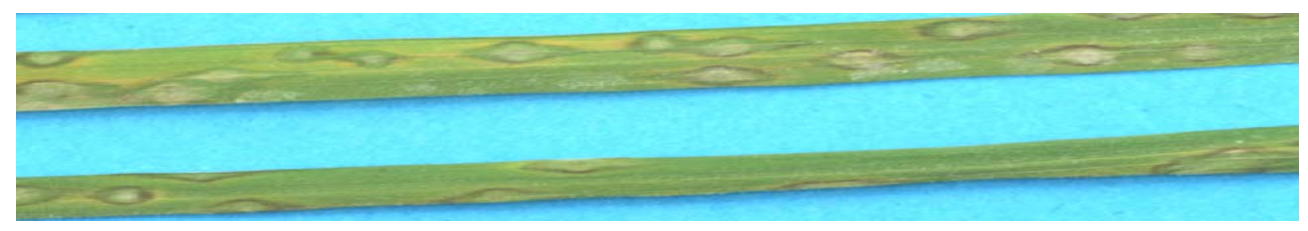

Maratelli (note de sévérité de la pyriculariose $=6$ )

Maratelli (blast severety note $=6$ )

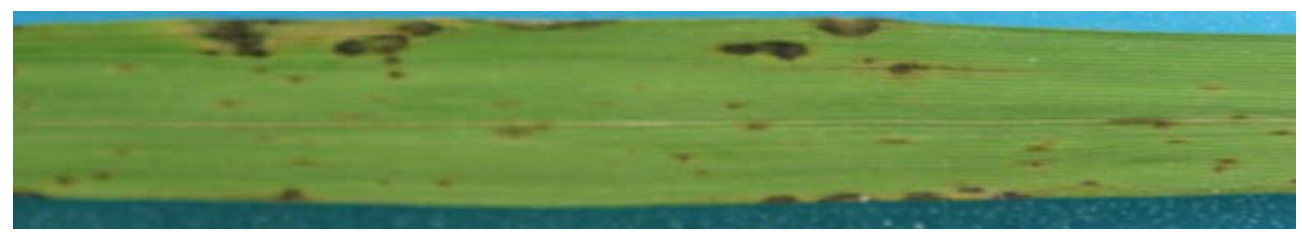

NERICA 2 (note de sévérité de la pyriculariose $=3$

NERICA 2 (blast severety note $=3$ )



NERICA 1 (note de sévérité de la pyriculariose $=5$ )

NERICA 1 (blast severety note $=5$ )

Figure 3 : Symptômes provoqués par la souche CD203 de Magnaporthe grisea sur les variétés de riz Maratelli, NERICA 2 et NERICA 1(Maratelli : très sensible ; NERICA 2 : résistant ; NERICA 1: sensible).

Symptoms of Magnaporthe grisea strain CD203 on rice varieties Maratelli, NERICA 2 and NERICA 1 (Maratelli : very sensitive ; NERICA 2 : resistant ; NERICA 1 : sensitive).

\section{DISCUSSION}

Dans l'ensemble, l'étude a mis en évidence un haut potentiel de résistance à la pyriculariose des variétés de riz NERICA 1 et NERICA 2 en Côte d'Ivoire. Cependant, le NERICA 1 s'est avéré sensible à certains isolats testés, contrairement à NERICA 2. Nous avions déjà noté dans la région du moyen Cavally notamment à Zouan (souspréfecture de Guiglo), une attaque relativement sévère (note de sévérité : 4 à 5) du NERICA 1 par la pyriculariose en 2002. Le bon niveau de résistance de NERICA 2 à la maladie, la place avec la variété Morobérékan (pluvial traditionnel de Côte d'Ivoire : Notteghem, 1981 ; Bouet et al., 2002), comme les riz pluviaux africains à haut potentiel de résistance à cette maladie en Côte d'Ivoire.
Il est probable d'après les réactions affichées, que la variété Bonfani soit munie d'une résistance fondamentalement complète à la pyriculariose quand, la variété Kéah exprime une résistance partielle et générale. La résistance générale qui a en plus, de fortes chances d'être durable (Vales, 1983). Aussi, la situation observée dans cette étude avec le Bonfani, poset-elle le problème de la relativité de la résistance déclarée d'une variété de riz à la pyriculariose. En effet, la résistance d'une variété donnée à la maladie est liée à l'échantillon d'isolats testés. II importe donc de savoir si la résistance variétale a été jugée par rapport à un isolat, à des isolats d'une zone géographique donnée ou à des isolats représentant la diversité dans la population du parasite. Dans le premier cas, il ne sera pas conseillé de diffuser la variété sauf si la population du parasite est caractérisée par un 
seul pathotype, ce qui est hypothétique en général. Dans le second cas, le développement du cultivar muni de la résistance ne pourrait dépasser les frontières de la zone d'étude. Pour le dernier cas de figure, le matériel végétal peut être cultivé à l'échelle nationale car, muni d'une résistance générale.

D'un point de vue pratique, bien que l'échantillon des isolats testés soit de taille réduite, les résultats enregistrés, permettent de rationaliser la gestion culturale des variétés NERICA 1 et NERICA 2 en Côte d'Ivoire. Cela, parce que cet échantillon représentait toute la diversité pathologique (groupes de races) et génétique (lignées clonales) de M. grisea, mise en évidence en Côte d'Ivoire (Bouet et al., 2002).

En effet, en plus de leur haut potentiel agronomique (Dingkuhn and al., 1997 ; Anonyme 2 , 2001), la résistance des variétés de riz NERICA1 et NERICA 2 aux maladies dont la pyriculariose, ont été des atouts majeurs exploités par les obtenteurs, pour convaincre les instances décisionnelles de la Côte d'Ivoire. Ces dernières ont en effet accepté (homologation) la culture de ces nouveaux riz africains dans le pays. La caution gouvernementale étant ainsi acquise, la diffusion des NERICA (1 et 2) a commencé effectivement vers la fin des années 90. Aujourd'hui, les variétés Bonfani et Kéah sont en train de gagner toutes les aires de culture de riz pluvial de la Côte d'Ivoire. Ainsi, dans les régions de l'ouest (Man, Danané, Biankouma), du centre-ouest (Daloa, Issia, Saïoua), du sudouest (San-pédro, Soubré), du sud-est (Tiassalé, N'Douci), du centre (Bouaké), du nord et nord-est (Korhogo, Odienné), des champs sont exploités. Face à cette conquête géographique progressive des NERICA, et d'après les résultats obtenus à l'issue de notre étude, des dispositions appropriées doivent être prises, à la fois, pour garantir une production optimale de ces variétés et maintenir très bas, le niveau de pression de la pyriculariose. Ainsi, une contribution de notre travail à cette entreprise est que, pour la variété NERICA 2, la diffusion peut se poursuivre si nécessaire à l'échelle régionale et nationale sans grand risque épidémique. En effet, cette variété a montré dans l'ensemble, une résistance générale à l'échantillon d'isolats testés.
Quant au NERICA 1, l'existence de souches de M. grisea qui lui sont compatibles (virulentes) peut compromettre son développement agricole. En effet, si le Bonfani est cultivé dans les zones de Man, de Waninou, de Touba, d'Odienné, de Séguela, de Boundiali et de Tiassalé, où, la probabilité de présence des isolats virulents est grande, une catastrophe agronomique et épidémique peut survenir. Au niveau agronomique, les plantes très affectées par la maladie, pourraient être détruites au stade végétatif avec comme conséquence une perte importante de production. Au niveau phytosanitaire, une explosion épidémique peut être engendrée du fait de la culture répétée du NERICA 1. Face à ces risques, il faut, à défaut de limiter la culture de NERICA 1 aux zones appropriées (probabilité nulle ou très faible d'existence de souches compatibles), exploiter cette variété en association ou en rotation avec le NERICA 2. Cela, pour maintenir l'épidémie à un seuil économiquement supportable par le riziculteur. Dans le cas échéant, il faudra préférer le NERICA 2 au NERICA 1. Cependant, bien que des risques existent, il faut relativiser nos propos car en milieu réel, les conditions à l'infection de la pyriculariose, ne sont pas aussi optimales comme celles créées au laboratoire.

\section{CONCLUSION}

Dans l'ensemble, l'étude a permis de mettre en évidence, un important potentiel de résistance à la pyriculariose des variétés de riz NERICA 1 et NERICA 2 en Côte d'Ivoire.

La diffusion en Côte d'Ivoire du NERICA 2 peut être poursuivie sans grand risque épidémique de la pyriculariose. Quant au NERICA 1, une approche plus raisonnée de vulgarisation doit être observée car, cette variété de riz peut être gravement attaquée par la pyriculariose dans certaines localités du pays. En perspective, il sera utile d'envisager une étude, pour apprécier les comportement des NERICA (1 et 2) face à la pyriculariose du cou, à travers des tests du pouvoir pathogène et/ou des essais multilocaux. Cette étude permettra de savoir, concernant spécifiquement le NERICA 2, si la variété est munie d'une protection complète (résistance à la pyriculariose foliaire et à la pyriculariose du cou) contre la pyriculariose. 


\section{REFERENCES}

Barr (M.E.). 1977. Magnaporthe, telimenella, and hyponectria, physosporellaceae. Mycologia, 69 : 952-966.

Bonman (M.J.), (B.A.) Estrada, and (J.M.) Bandong. 1989. Leaf and neck blast resistance in tropical lowland rice cultivars. Plant disease vol. $73 \mathrm{~N}^{\circ} 5: 388-390$.

Bouet (A.), (J.) Millazo, (H.) Adreit, (J.L.) Notteghem, (D.) Tharreau. 2002. Mise en évidence de races et de lignées clonales dans la population de Magnaporthe grisea de Côte d'Ivoire. Agronomie Africaine XIV (1) : 5169.

Dingkuhn (M.), (M.P.) Jones, (D.E.) Johnson, (B.) Fofana and (A.) Sow. 1997. Oryza sativa and 0 . glaberrima genepools for high yielding, weed-competitive rice plant types. In : S. Fukai, M. Cooper and J. Salisbury (ed.), Breeding strategies for rainfed lowland rice in drought-prone environment. International workshop of the rainfed lowland rice consortium, December 1996, Ubon, Thaïland. ACIAR proceeding $\mathrm{N}^{\circ} 77$, Australian Center of International Agricultural Research (ACIAR), Canberra, Australia, pp.144-155.

Johnson (D.E.), (M.P.) Jones, (M.) Dingkuhn and (M.C.) Mahamane. 1998. Weed competitive rice plants from the $O$. sativa and $O$. glaberrima genepools for improved weed management in the rainfed rice system of west Africa. In : Proceedings of the 17th COLUMA Conference, Dijon, December 1998. ANPP, Paris, Vol. 1, pp. 71-78.

Notteghem (J.L.). 1981. Analyse des résultats d'inoculation de 67 variétés de riz par 15 isolats de Pyricularia oryzae. In : Symposium sur la résistance du riz à la pyriculariose, IRAT/GERDAT, Montpellier, France : 74-95.

Plowright (R.A.), (D.L.) Coyne, (P.) Nash and (M.P.) Jones. 1999. Resistance of the rice nematod Heterodera sacchari, Meloidogyne graminicola and $M$. incognita in Oryza glaberrima and $O$. glaberrima $X O$. sativa interspecific hybrids. Nematology 1 (7-8) : 745-751.

Vales (M.). 1983. Des connaissances sur les relations hôtes parasite aux stratégies de lutte contre la pyriculariose du riz. Thèse de 3 ème cycle. université Paris-sud, centre Orsay, $310 \mathrm{p}$.

Anonyme 1. 1998. New Rice for Africa. WARDA, Bouaké, Côte d'Ivoire, $20 \mathrm{p}$.

Anonyme 2. 2001. Bintou and her New Rice for Africa. WARDA (West Africa Rice Development Association), Bouaké, Côte d'Ivoire, 32 PP. 